\title{
Object-oriented segmentation of remote sensing image based on texture analysis
}

\author{
Yanhong Wang \\ Institute of Remote Sensing and Digital Earth \\ Chinese Academy of Sciences \\ University of Chinese Academy of Sciences \\ Beijing, China \\ Wangyanhong321@126.com
}

\author{
Bo Cheng ${ }^{1}$, Guizhou Wang ${ }^{1,2}$, Shucheng You ${ }^{3}$ \\ 1.Institute of Remote Sensing and Digital Earth \\ Chinese Academy of Sciences \\ 2.University of Chinese Academy of Sciences \\ 3.China Land Surveying \& Planning Institute \\ Beijing, China \\ chengbo@ceode.ac.cn
}

\begin{abstract}
High-spatial-resolution remote sensing image contains rich texture information and high variability of spectral information, but the rich details greatly increase the difficulty of segmentation. For this reason, this paper proposes a new method of high-spatial-resolution remote sensing image segmentation based on texture features. First, texture features of high-spatialresolution remote sensing image were extracted by grey level cooccurrence probability (GLCP) method. Then the texture and spectral features are separately used for segmentation in eCognition. By using the texture features to segment the image, the segmentation is more accurate and smoother and has less broken region. The advantage of the method based on texture features is evaluated by comparison of traditional method based on spectral information. It shows that the new method is more reliable and comprehensive in reflecting the ground objects in the image.
\end{abstract}

Keywords - Texture feature, High-resolution, Object-oriented, Image segmentation.

\section{INTRODUCTION}

Segmentation of remote sensing image is a fundamental process in image processing. It is the basis of object-oriented processing of remote sensing image and plays an important role in the theoretical research of object-oriented image analysis. With the development of remote sensing technology and the improvement of the satellite spatial resolution, highresolution images have been widely used in various fields. Though it provides high spatial resolution, rich texture information and multi-spectral information, the rich details greatly increase the difficulty of segmentation. For this reason, the segmentation of high resolution image becomes one of the main issues.

Texture is a significant property in digital imagery. It plays an important role in human visual perception and offers information for recognition and interpretation. On account of the high variability of spectral information in high resolution remote sensing image, the traditional segmentation method which is based on merely spectral character can't meet the demand of high resolution image. Selecting texture features which are independent and discriminable will aid in the segmentation process ${ }^{[1]}$.

In this study, we compute a set of grey spatialdependence probability-distribution matrices for a given image block and extract a set of 8 texture features form each of these matrices. These features contain information about such image textural characteristics as homogeneity, linear structure, contrast, number and nature of boundaries present, and the complexity of the image. We also investigate the usefulness of texture features ${ }^{[2 \sim 4]}$ for segmentation.

This paper is organized as follows: In Section II, the theory of texture analysis is briefly reviewed. In Section III the segmentation experimental results based on various texture features and spectral features are discussed in detail. Finally, conclusion is given in SectionIV.

\section{TEXTURE ANALYSIS}

Texture analysis is a process that extract texture feature through a certain image processing technology, thereby obtaining the quantification or qualitative description of texture. Tuceryan and Jain categorize the various texture analysis methods into four groups: statistical, geometrical, model-based and signal processing-based ${ }^{[5,6]}$. The grey level co-occurrence probability (GLCP) method that belongs to statistical analysis method is a common algorithm of generating texture features, and has strong adaptability and robustness ${ }^{[7]}$. As a traditional method of image texture analysis, it has been widely used in digital image processing.

The GLCPs provide a second-order method for generating texture features. Given a spatial window within the image, the GLCPs represent the conditional joint probabilities of all pair-wise combinations of grey levels given two parameters: inter-pixel distance $(\delta)$ and orientation $(\theta)$. It should be noted that since the image window is a discrete set, it is convenient to represent the parameters $\delta$ and $\theta$ as cartesian co-ordinates $\delta x$ and $\delta y$ which represent the interpixel spacings in the $\mathrm{x}$ - and $\mathrm{y}$-directions respectively. The probability measure can be defined as:

$$
\operatorname{Pr}(x)=\left\{C_{i j} \mid(\delta x, \delta y)\right\}
$$

where $C_{i j}$ (the co-occurrence probability between grey levels i and $\mathrm{j}$ ) is defined as:

$$
C_{i j}=\frac{P_{i j}}{\sum_{i, i=1}^{G} P_{i j}}
$$

where $P_{i j}$ represents the frequency of occurrence between two grey levels, $\mathrm{i}$ and $\mathrm{j}$, for a given displacement vector $(\delta x, \delta y)$ for 
the specified window size. $G$ is the number of quantized grey levels for the entire image. The sum in the denominator represents the total possible number of grey level pairs $(i, j)$ within the window, given $(\delta x, \delta y)$.

Statistics are applied to the GLCPs to generate texture features. Although many texture statistics can be applied, eight common grey level shift invariant statistics are considered (Table I).

TABLE I. GREY LEVEL SHIFT-INVARIANT COOCCURRENCE TEXTURE STATISTICS

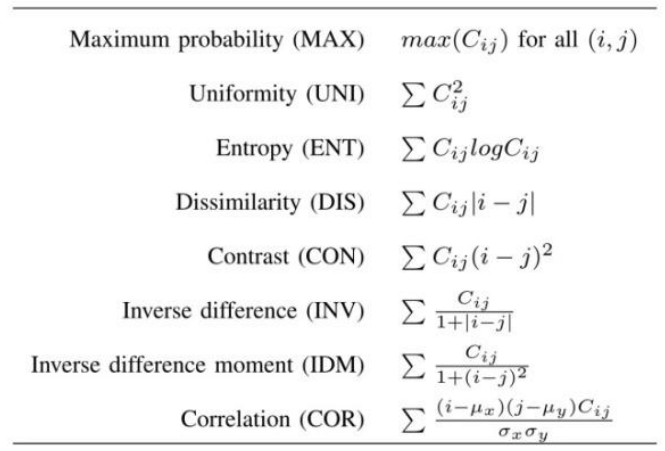

\section{RESULTS AND ANALYSIS}

\section{A. Test data}

The study area is located in northwest of Anshan city in Liaoning province. We select the ALOS imagery (containing four multispectral bands and one panchromatic band), acquired in May, 2010, as experiment data. We fuse the multispectral and panchromatic bands using the orthographic image. A $600 \times 600$ pixel subset of the fusion imagery (resolution is $2.5 \mathrm{~m}$ with 4 bands) is then selected for analysis.

\section{B. Test 1 select the usefulness texture features}

1) Selecting the band which contains the richest information

Covariance is the symbol of the amount of information that the bands contained. The lager the covariance is, the more information the band contains. In order to avoid the redundancy of the band, the band with maximum information is selected by calculating the statistical covariance matrix of each band.

TABLE II THE STATISTICS COVARIANCE MATRIX OF EACH BAND OF ALOS

\begin{tabular}{|c|c|c|c|c|}
\hline Covariance & Band 1 & Band 2 & Band 3 & Band 4 \\
\hline Band 1 & 288.682 & 359.941 & 461.940 & 354.547 \\
\hline Band 2 & 359.941 & 461.612 & 601.614 & 457.844 \\
\hline Band 3 & 461.940 & 601.614 & 804.768 & 585.083 \\
\hline Band 4 & 354.547 & 457.844 & 585.083 & 536.510 \\
\hline
\end{tabular}

Table II shows the statistical covariance matrix of each band of ALOS imagery. Variance in descending order is Band3, Band4, Band2, Band1, therefore, Band3 contains the richest information.

2) Texture features generation

This study extract a set of eight texture statistics from the co-occurrence matrix of Band3, which are mean、variance、 uniformity、contrast、 dissimilarity 、 entro py , inverse difference and correlation. We compare the eight characters to select the salient texture feature. The parameters set as: the processing window size is $3 \times 3, d=1, \theta=45^{\circ}$ and the grayscale quantization level is 64 . At last, consider mean and variance as the most significant texture statics for segmentation.

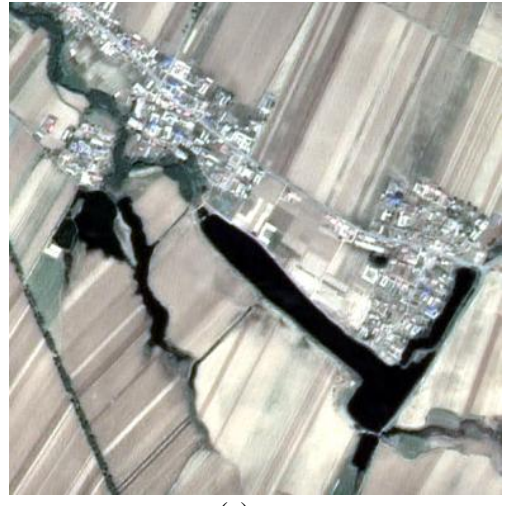

(a)

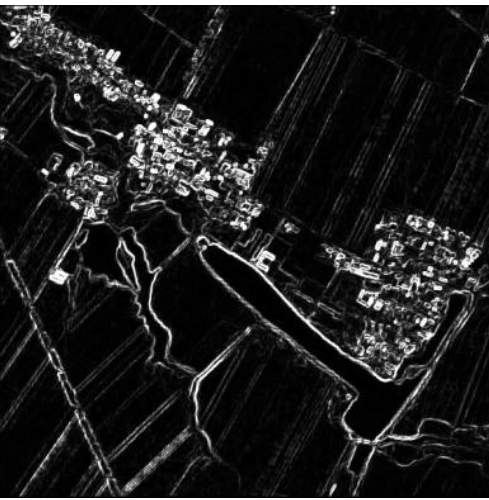

(b)

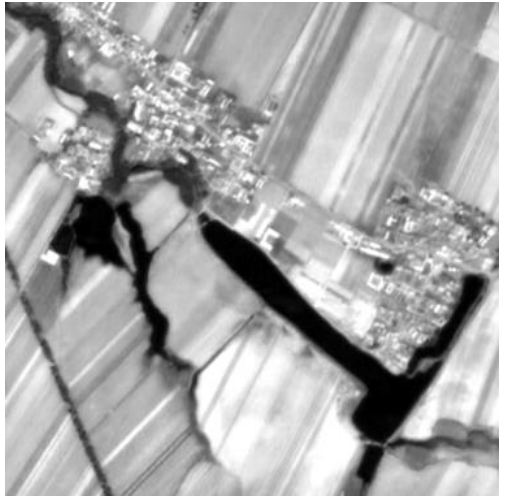

(c)

Fig. 1. Different statistic texture feature maps. Original image included. (a) Original image, (b) Variance texture statics, (c) Mean texture statics

\section{Test2 segmentation of high resolution image}

Then we used the selected texture features for segmentation in eCognition. In this section we present results of our studies on the usefulness of the texture statics for segmentation. Four methods are used in our study, which is the segmentation based on spectral feature, the variance texture statics, mean texture statics, and the combine of the texture statics of variance and mean. And the segmentation parameter set as scale 20 , shape index 0.2 , impact 0.5 .

It is obvious that the results using traditional method (a) is too broken and the phenomenon of over-segmentation is serious. Most of the surface features can be divided correctly in (b)、(c). They also do a good job in details. Compare to the results in (a), (b) and (c) avoid the phenomenon of over-segmentation, to some extent, and the segmentation boundary is more accurate and smoother. 
Further observation, we can find that (b)makes a good performance for the regions with grey uniformity and the objects with small variance value (such as filed, water). However, for the objects with large variance value (such as building land), (c) shows a better effect than (b). According to that, this study uses the combination of the texture statics of mean and variance to segment the image. It shows the segmentation result (d) combine the advantage of (b) and (c). At last, we attempt to segment the image based on the spectral feature and the selected texture features, the results shows in (e), it is superior to (a), but still have many broken regions, and it is unsatisfactory.

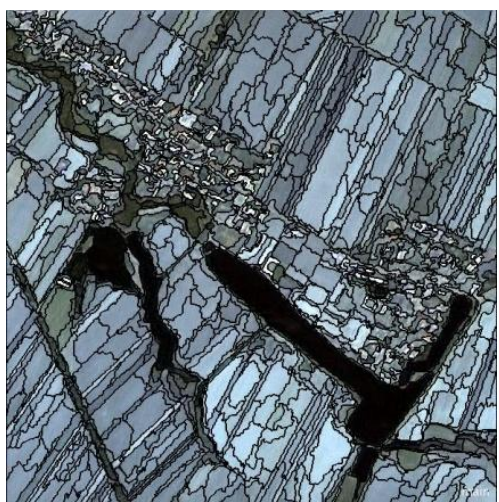

(a)

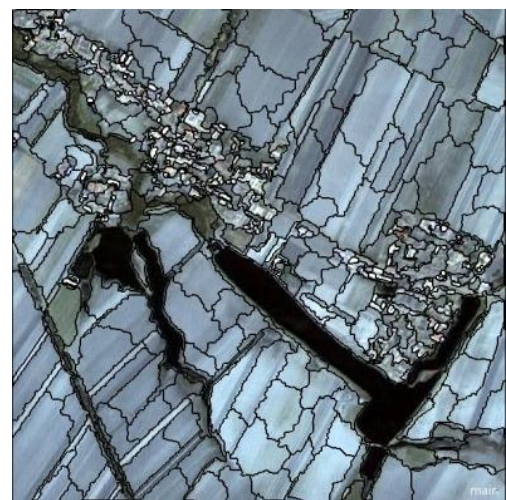

(b)

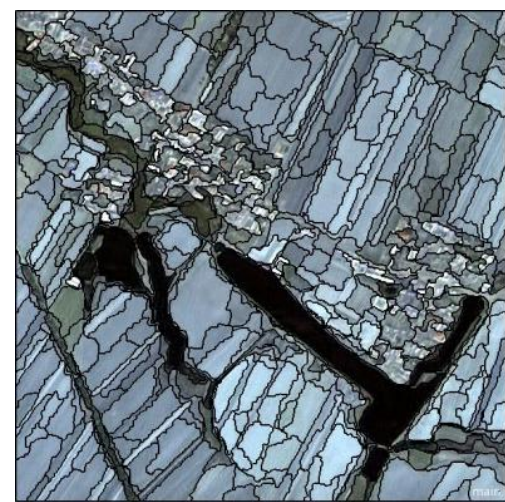

(c)

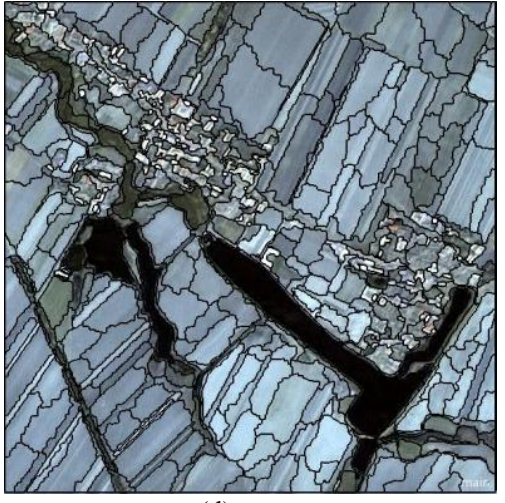

(d)

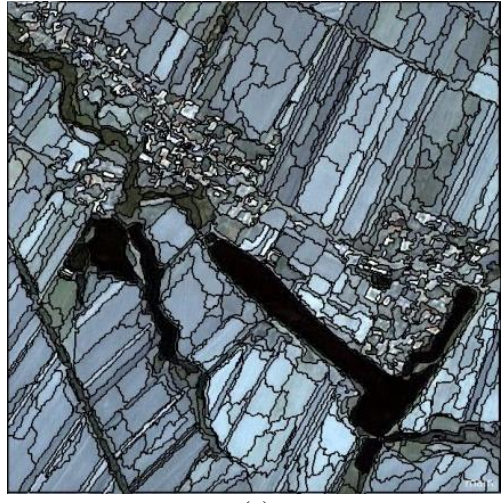

(e)

Fig.2. Segmentation results based on different algorithms. (a) the result using traditional method(based on spectral feature), (b) the result based on variance texture statics, (c) the result based on the mean texture statics, (d)the results based on the combination of the texture statics of variance and mean(weight $1: 1)$, (e)the results based on the combination of the texture features and spectral feature.

The statics of the number of pixels within region of the five methods is showed in Fig. 3. We choose the region with the number of pixels which is less than 200 as the criteria to measure the degree of the fragmentation. In (a) and (e), it accounts for $42.0 \%$ and $35.4 \%$ individually, which indicates the results of the segmentation is too broken. In comparison, it accounts for less than $20 \%$ in both (b) and (c). It is a little lager in (e), which accounts for $30.1 \%$. On the whole, the methods based on texture features avoid the phenomenon of over-segmentation effectively.

For this research, the results indicate the method based on texture analysis is appropriate for the segmentation of high resolution remote sensing image. It can gasp the surface features well, not only the overall structure, but also the details of the image. Given the results for the Fig2, it is demonstrated that the method consider the texture feature is an improvement over the traditional improves the segmentation.

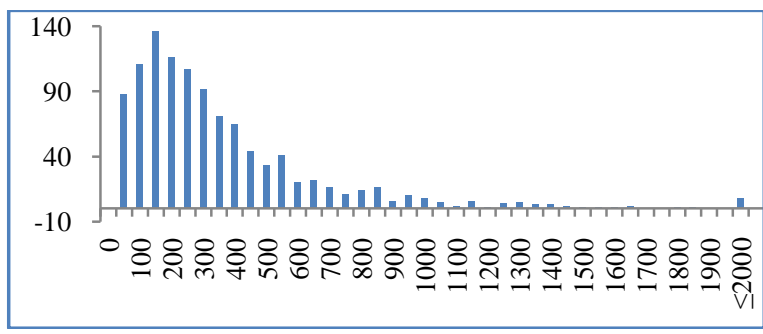

(a)

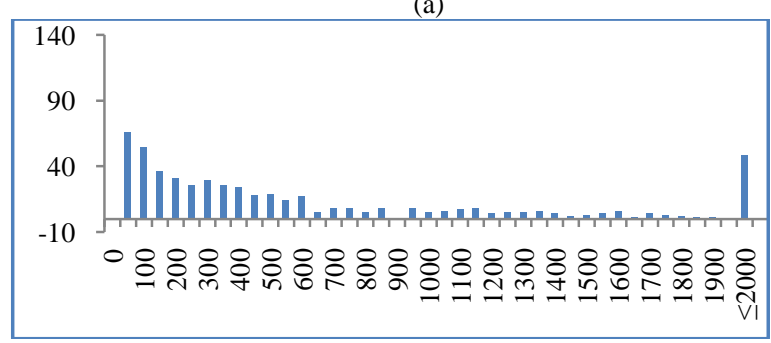

(b) 


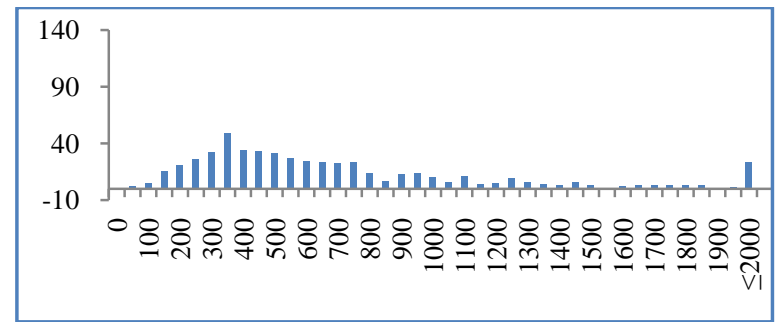

(c)

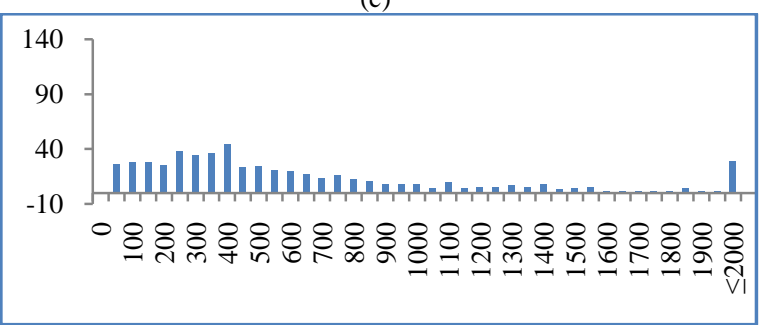

(d)

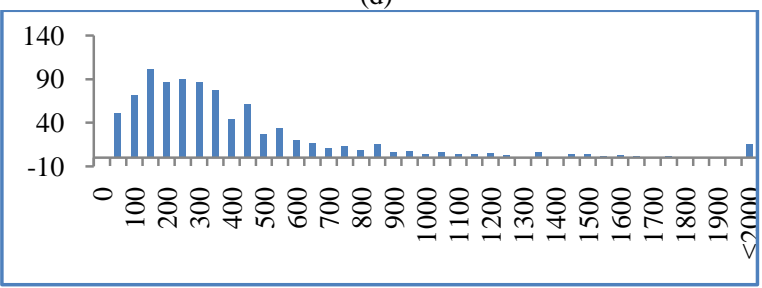

(e)

Fig. 2. The statics of the number of pixels within region of the methods (a) $\sim(\mathrm{e})$.

\section{Segmentation evaluation}

Most evaluation proposed so far can be categorized into direct methods and indirect methods. The direct methods study segmentation algorithms themselves while indirect methods evaluate the performance of algorithms according to the quality of segmented images. In this research, we use indirect methods for qualitative evaluating the results of the segmentation, and the specific evaluation criteria are uniformity within region (UM) 、 contrast across region (GC) 、 variance contrast across region (VC) and edge gradient measure (EG).

TABLE III. THE VALUE FOR MEASURE FOR EVALUATION

\begin{tabular}{|c|c|c|c|c|c|}
\hline Methods & $\begin{array}{c}\text { UM (we } \\
\text { ight } \\
\mathbf{0 . 2} \text { ) }\end{array}$ & $\begin{array}{c}\text { GC } \\
\text { (weight } \\
\mathbf{0 . 3}\end{array}$ & $\begin{array}{c}\text { VC } \\
\text { (weight } \\
\mathbf{0 . 2} \text { ) }\end{array}$ & $\begin{array}{c}\text { EG } \\
\text { (weight } \\
\mathbf{0 . 3} \text { ) }\end{array}$ & Score \\
\hline (a) & 0.298 & 0.437 & 0.449 & 0.400 & 0.400 \\
\hline (b) & 0.265 & 0.661 & 0.479 & 0.411 & 0.470 \\
\hline (c) & 0.656 & 0.720 & 0.724 & 0.640 & 0.684 \\
\hline (d) & 0.531 & 0.591 & 0.570 & 0.479 & 0.541 \\
\hline (e) & 0.359 & 0.287 & 0.672 & 0.449 & 0.427 \\
\hline
\end{tabular}

Tab.III shows the value of segmentation evaluation using four methods. For both (c) and (d), it is very apparent that they are superior to the traditional method (method a) in the four evaluation criteria. For the reason that the object with different spectrum feature is merged into one region in method (b), the uniformity with region of (b) is slightly inferior to (a), but it doesn't influence the entire performance. Obviously, (e) is inferior to (b)、 (c)、(d).

\section{CONCLUSION}

For this research, we extract the texture features by grey level co-occurrence probability (GLCP) method, and then investigate the usefulness of texture features for segmentation in eCognition. The advantage of the method based on texture features is evaluated by comparison of traditional method based on spectral information. It shows the new method is more accurate and reliable than traditional method.

Image segmentation has become essential for high resolution remote sensing imagery. The further development of first promising segmentation approaches offers a lot of potentials to make remote sensing image analysis more accurate as well as more efficient. The use of texture information for segmentation could improve the results. Now it is about a set of 28 texture features, further study is needed on how to combine the texture information for segmentation to obtain the optimal results.

\section{ACKNOWLEDGMENT}

The research has been supported by International Science \& Technology Cooperation Program of China

(2012DFA20930) and the grants from the union project of Director Foundation of Centre for Earth Observation and Digital Earth, Chinese Academy of Sciences.

\section{REFERENCES}

[1] R. Jobanputra and D. A. Clausi, "Preserving boundaries for image texture segmentation using grey level co-occurring probabilities," Pattern Recognition, vol. 39, pp. 234-245, 2006.

[2] D.G. Barber and E.F. LeDrew, "SAR sea ice discrimination using texture statistics: a multivariate approach, " Photogrammetric Engineering and Remote Sensing, vol.57, pp. 385-395, 1991.

[3] A. Baraldi and F. Parmiggiani, "An investigation of the textural characteristics associated with gray level co-occurrence matrix statistical parameters, "IEEE Transactions on Geoscience and Remote Sensing, vol. 33, pp.293-303, 1995.

[4] D. A. Clausi, "An analysis of co-occurrence texture statistics as a function of grey level quantization, " Canadian Journal of Remote Sensing, vol.28, pp. 45-62,2002

[5]H. Zhang, J. E. Fritts, and S. A. Goldman, "Image segmentation evaluation: A survey of unsupervised methods," Computer Vision and Image Understanding, vol. 110, pp. 260-280, 2008.

[6] D. A. Clausi, "An analysis of co-occurrence texture statistics as a function of grey level quantization," Canadian Journal of remote sensing, 2002, pp.45-62.

[7] M. Tuceryan and A.K. Jain, "Handbook of pattern Recognition and computer Vision," Chapter 2: Texture Analysis, World Scientific, Singapore, 1993 